\title{
Schwinger model on a half-line
}

\author{
Yeong-Chuan Kao and Yu-Wen Lee \\ Department of Physics, National Taiwan University, Taipei, Taiwan
}

(Received 7 August 2001; published 8 February 2002)

\begin{abstract}
We study the Schwinger model on a half-line in this paper. In particular, we investigate the behavior of the chiral condensate near the edge of the line. The effect of the chosen boundary condition is emphasized. The extension to the finite temperature case is straightforward in our approach.
\end{abstract}

DOI: 10.1103/PhysRevD.65.067701

PACS number(s): $11 \cdot 10 . \mathrm{Kk}$

\section{INTRODUCTION}

The (1+1)-dimensional massless spinor electrodynamics, the Schwinger model [1], has been a most popular playground for theorists because it exhibits many subtle properties which are believed to exist, yet difficult to verify exactly, in four-dimensional QCD [2-4]. One such property is the existence of the chiral condensate, an essential component in modern particle physics. In the Schwinger model, a rather complete understanding of the chiral condensate under various circumstances, e.g., at finite temperature and finite chemical potential [5-10], has been achieved. It is the unrealistic low dimensionality of the model that allows such detailed understanding. But sometimes realistic lowdimensional models can arise from $(3+1)$-dimensional ones if only radial dependence ( $s$-wave approximation) is kept. For example, the famous Callan-Rubakov effect, i.e., the monopole catalysis of proton decay [11-13], made use of a Schwinger-like model defined on a half-line in the first approximation. A fermion number breaking condensate analogous to the chiral condensate can be shown to exist around a magnetic monopole.

Motivated partly by the Callan-Rubakov effect, we study the original Schwinger model on a half-line. We pay special attention to the dependence of the chiral condensate on the distance from the edge. Exact results can be obtained by using existing techniques. The standard value of the chiral condensate is recovered when we are in a region far away from the edge. The value of the condensate near the edge depends on the chosen boundary condition. In this paper we adopt the boundary condition employed in the boundary conformal field theory approach to the Callan-Rubakov effect. It is easy to extend the result to the finite temperature case with our approach. Previous attempts to study the finite temperature behavior of the Callan-Rubakov condensate relied on the cluster decomposition property which is hard to implement on a half line [14]. A recent work by Dürr [15], building on and correcting ealier results by Dürr and Wipf $[15,16]$, has studied the behavior of the chiral condensate in the Schwinger model on a finite segment with boundary conditions similar to ours. Dürr's result is the same as ours in regions where comparisons can be made, yet his approach and emphasis is rather different from those adopted here.

\section{BOSONIZATION OF QED $_{2}$ ON A HALF-LINE}

We shall begin by first studying the Schwinger model on a finite segment. The formulation we have followed is rather standard [4]. The Lagrangian density of $\mathrm{QED}_{2}$ on a line segment of length $L(x \in[0, L])$ is defined by

$$
\mathcal{L}=-\frac{1}{4} F_{\mu \nu}^{2}+\bar{\psi} \gamma_{\mu}\left(i \partial_{u}-e A_{\mu}\right) \psi
$$

The boundary condition on $\psi$ is chosen to be $\psi_{R}(t, L)$ $+\psi_{L}(t, L)=0=\psi_{R}(t, 0)+\psi_{L}(t, 0)$ which breaks chiral symmetry at $x=0$ and $L$ [13]. A similar boundary condition appears in the Callan-Rubakov effect and allows an exact treatment of the theory. Discussions on a generalization of this boundary condition will be given in Sec. IV.

The half-line case will be obtained if we take the infinite $L$ limit at the end of the analysis. In two dimensions, it is possible to choose the Coulomb gauge so that $A_{x}$ is independent of the spatial coordinate $x$, i.e. $\partial_{x} A_{x}=0$. In the Coulomb gauge, the time component of the gauge field $A_{0}$ is obtained as $A_{0}(t, x)=-e \int_{0}^{L} d y D(x, y) j^{0}(t, y)$ by solving the Gauss's law

$$
E^{\prime}=e j^{0}, \quad \dot{E}=-e j^{1} .
$$

$D(x, y)$ is the Green's function which satisfies $\partial_{x}^{2} D(x, y)$ $=\delta(x-y)$. To further our analysis, we will restrict ourselves in the charge zero sector, i.e. $Q \equiv \int_{0}^{L} d x j_{0}(x)=0$. This immediately implies the boundary condition for the electric field: $E(0)=E(L)$. The form of the Green function $D(x, y)$ obeying the restriction is found to be

$$
D(x, y ; \lambda)=\frac{1}{2}|x-y|+a(x+y)+c
$$

if we require $A_{0}(x)$ to obey the following boundary condition : $(d / d x) A_{0}(0, t)=0=(d / d x) A_{0}(L, t)[17]$. Here $a$ and $c$ are arbitrary constant.

We define $(e L / 2 \pi) A_{x}=W(t)$, the Wilson line operator, which will be needed in later formulations. It is perhaps worthwhile to emphasize that as our theory is not defined on a torus (our boundary conditions are "open"), the winding number for the gauge field is not defined and the Wilson line operator is not an angular variable, due to the absence of topologically nontrivial gauge transformations [16].

Following standard procedures, the Hamiltonian reads 


$$
\begin{aligned}
H= & \frac{F^{2}}{2 L}+\int_{0}^{L}\left[\psi_{R}^{\dagger} i \partial_{x} \psi_{R}(x)-\psi_{L}^{\dagger} i \partial_{x} \psi_{L}(x)\right] \\
& +\frac{2 \pi}{L} W(t) \int_{0}^{L} d x\left[\psi_{R}^{\dagger} \psi_{R}(x)-\psi_{L}^{\dagger} \psi_{L}(x)\right] \\
& -\frac{e^{2}}{2} \int_{0}^{L} d x \int_{0}^{L} d y j^{0}(t, x) D(x, y) j^{0}(t, y),
\end{aligned}
$$

where $j^{0}(t, x) \equiv \psi_{R}^{\dagger} \psi_{R}(x)+\psi_{L}^{\dagger} \psi_{L}(x)$. The fermion field is written in terms of right- and left-moving components explicitly for later convenience.

The Hamiltonian (4) is essentially a model of fermions interacting via long-range Coulomb forces together with the quantum mechanical degrees of freedom of the spatial component of the gauge field. We will empoly the methods developed for boundary critical phenomena $[18,19]$.

The first step of bosonizing $[9,20]$ the fermions with the chosen boundary conditions at the origin is mirror copying: our boundary condition $\psi_{R}(t, 0)=-\psi_{L}(t, 0)$ allows us to analytically continue the left-moving component of the fermion field to the domain $x<0$ by defining $\psi_{R}(t, x) \equiv$ $-\psi_{L}(t,-x)$ for $x \in[-L, 0]$. Our theory can then be formulated as a theory of a chiral (right-moving) fermion defined on the full segment $(x \in[-L, L])$ which leads to the full line in the infinite $L$ limit. Our Hamiltonian then reads as

$$
\begin{aligned}
H= & \frac{F^{2}}{2 L}+\int_{-L}^{L} d x \psi_{R}^{\dagger} i \partial_{x} \psi_{R}(x) \\
& +\frac{2 \pi}{L} W(t) \int_{-L}^{L} d x \operatorname{sgn}(x) \rho_{R}(x)-\frac{e^{2}}{2} \int_{0}^{L} d x \int_{0}^{L} d y\left[\rho_{R}(x)\right. \\
& \left.+\rho_{R}(-x)\right] D(x, y)\left[\rho_{R}(y)+\rho_{R}(-y)\right]
\end{aligned}
$$

where $\rho_{R}(x) \equiv \psi_{R}^{\dagger}(x) \psi_{R}(x)$. We now bosonize the Hamiltonian by introducing a single chiral boson field $\hat{\phi}(x)$ with $\psi_{R}(x)=1 / \sqrt{2 L}: e^{i \hat{\phi}(x)}:=(1 / \sqrt{2 \pi a}) e^{i \hat{\phi}(x)}$, where $a$ is an ultraviolet cutoff $[9,20]$. Notice that our boundary condition on $x=L, \psi_{R}(t, L)=-\psi_{L}(t, L)$, now implies that we have the periodic boundary conditions for the chiral fermion, i.e., $\psi_{R}(L)=\psi_{R}(-L)$. The chiral boson therefore has the following mode expansion:

$$
\begin{aligned}
& \hat{\phi}(t, x)=q_{R}+\frac{2 \pi p_{R}(t-x)}{2 L}+\phi(t, x), \\
& \phi(t, x)=\sum_{q>0} \sqrt{\frac{\pi}{q L}}\left(e^{i q x-a q / 2} b_{q}+\text { H.c. }\right),
\end{aligned}
$$

$q=2 \pi n / 2 L$, and the boson creation operators satisfy the usual commutation relations $\left[b_{q}, b_{q^{\prime}}^{\dagger}\right]=\delta_{q, q^{\prime}},\left[q_{R}, p_{R}\right]=i$. The periodic boundary condition requires $p_{R}$ to take integer values only. The meaning of various factors in the above formulas can be seen from the following bosonization identity:

$$
\begin{aligned}
& \psi_{R}^{\dagger}(x) \psi_{R}(x)=\frac{1}{2 \pi} \partial_{x} \hat{\phi}(x)=\frac{p_{R}}{2 L}+\frac{\partial_{x} \phi(x)}{2 \pi}, \\
& \psi_{L}^{\dagger}(x) \psi_{L}(x)=\frac{-1}{2 \pi} \partial_{x} \hat{\phi}(-x)=\frac{p_{R}}{2 L}-\frac{\partial_{x} \phi(-x)}{2 \pi},
\end{aligned}
$$

so that $p_{R}=\int_{0}^{L} d x\left(\psi_{R}^{\dagger} \psi+\psi_{L}^{\dagger} \psi_{L}\right)$ is the charge operator while the chiral charge operator is $\int_{0}^{L} d x\left(\psi_{R}^{\dagger} \psi_{R}-\psi_{L}^{\dagger} \psi_{L}\right)=(1 / \pi)$ $\times[\phi(L)-\phi(0)] \equiv q_{5}$. Notice that the chiral charge operator here appears as a surface term rather than the zero mode of Dirac fermions. This is because the theory is defined on a segment rather on a torus.

Some straightforward manipulations bring us the bosonized kinetic energy term

$$
H_{F}=\frac{1}{4 \pi} \int_{-L}^{L} d x\left(\partial_{x} \phi+\frac{2 \pi p_{R}}{2 L}+\frac{2 \pi}{L} W \operatorname{sgn}(x)\right)^{2} .
$$

While for the interaction term, we have

$$
\begin{aligned}
H_{\text {int }} & =\frac{e^{2}}{8 \pi^{2}} \int_{0}^{L} d x[\phi(x)-\phi(-x)]^{2} \\
& =\frac{e^{2}}{8 \pi^{2}} \int_{-L}^{L} d x\left[\phi(x)^{2}-\phi(x) \phi(-x)\right],
\end{aligned}
$$

which is manifestly gauge invariant.

Since we have restricted ourselves to the charge zero sector, $p_{R}$ is set to zero. Setting everything together, we finally arrive at the following bosonized Hamiltonian:

$$
\begin{aligned}
H= & \frac{F^{2}}{2 L}+\frac{2 \pi Q_{5}^{2}}{L}+\frac{1}{4 \pi} \int_{-L}^{L} d x\left[\left[\partial_{x} \phi(x)\right]^{2}\right. \\
& \left.+\frac{m^{2}}{2}\left[\phi(x)^{2}-\phi(x) \phi(-x)\right]\right] \\
= & \frac{F^{2}}{2 L}+\frac{2 \pi Q_{5}^{2}}{L}+\sum_{q>0} \frac{m^{2} / 2+q^{2}}{q} \\
& \times\left[b_{q}^{\dagger} b_{q}-\frac{m^{2} / 2}{2\left(m^{2} / 2+q^{2}\right)}\left(b_{q} b_{q}+b_{q}^{\dagger} b_{q}^{\dagger}\right)\right],
\end{aligned}
$$

where $m^{2}=e^{2} / \pi$ and $Q_{5} \equiv 2 W-q_{5}$. As the Hamiltonian contains only quadratic terms, we can solve for the ground state wave functional exactly. Terms of order of $O(1 / L)$ in the Hamiltonian will be neglected from now on as they will not influence the final result. We therefore concentrate on terms propotional to $b_{q} b_{q}, b_{q}^{\dagger} b_{q}^{\dagger}$, or $b_{q}^{\dagger} b_{q}$. This part of the Hamiltonian can be diagonalized easily via a Bogoliubov transformation

$b_{q} \equiv \cosh \varphi_{q} \hat{b}_{q}-\sinh \varphi_{q} \hat{b}_{q}^{\dagger} \quad$ with $\quad \tanh 2 \varphi_{q}=\frac{-m^{2} / 2}{m^{2} / 2+2 q^{2}}$

The diagonalized Hamiltonian now reads 


$$
H=\sum_{q>0} \sqrt{m^{2}+q^{2}} \hat{b}_{q}^{\dagger} \hat{b}_{q}
$$

This shows clearly the well known fact that the spectrum contains a relativistic boson with mass $m$. The spectrum contains only a positive momentum branch reflecting the fact that $\phi$ is a chiral boson. With this simple bosonic Hamiltonian and the bosonic representations of fermionic operators, we can easily calculate the fermion correlation functions.

\section{CHIRAL CONDENSATE}

The chiral condensate is given by $\bar{\psi}(x) \psi(x)$ $=\psi_{R}^{\dagger}(x) \psi_{L}(x)+$ H.c. When written in terms of the chiral bosonic field it becomes

$$
\begin{aligned}
\psi_{R}^{\dagger}(x) \psi_{L}(x)= & -\psi_{R}^{\dagger}(x) \psi_{R}(-x) \\
= & \frac{-1}{2 \pi a} e^{-i \hat{\phi}(x)} e^{i \hat{\phi}(-x)}=\frac{-1}{2 \pi a}: e^{-i \phi(x)+i \phi(-x)}: \\
& \times e^{\langle 0|\phi(x) \phi(-x)-(1 / 2)[\phi(x) \phi(x)+\phi(-x) \phi(-x)]| 0\rangle} .
\end{aligned}
$$

To proceed, we need the following relations from Eq. (11):

$$
\begin{aligned}
\left\langle 0\left|b_{q} b_{q^{\prime}}\right| 0\right\rangle= & -\cosh \varphi_{q} \sinh \varphi_{q^{\prime}}\left\langle 0\left|\hat{b}_{q^{\prime}} \hat{b}_{q^{\prime}}^{\dagger}\right| 0\right\rangle \\
& -\sinh \varphi_{q} \cosh \varphi_{q^{\prime}}\left\langle 0\left|\hat{b}_{q}^{\dagger} \hat{b}_{q^{\prime}}\right| 0\right\rangle, \\
\left\langle 0\left|b_{q} b_{q^{\prime}}^{\dagger}\right| 0\right\rangle= & \cosh \varphi_{q} \cosh \varphi_{q^{\prime}}\left\langle 0\left|\hat{b}_{q^{\prime}} \hat{b}_{q^{\prime}}^{\dagger}\right| 0\right\rangle \\
& +\sinh \varphi_{q} \sinh \varphi_{q^{\prime}}\left\langle 0\left|\hat{b}_{q}^{\dagger} \hat{b}_{q^{\prime}}\right| 0\right\rangle, \\
\left\langle 0\left|b_{q}^{\dagger} b_{q^{\prime}}\right| 0\right\rangle= & \sinh \varphi_{q} \sinh \varphi_{q^{\prime}}\left\langle 0\left|\hat{b}_{q} \hat{b}_{q^{\prime}}^{\dagger}\right| 0\right\rangle \\
& +\cosh \varphi_{q} \cosh \varphi_{q^{\prime}}\left\langle 0\left|\hat{b}_{q^{\dagger}}^{\dagger} \hat{b}_{q^{\prime}}\right| 0\right\rangle .
\end{aligned}
$$

With the above relations, we obtain

$$
\begin{aligned}
D_{1}(x)= & \langle 0|\phi(x) \phi(-x)| 0\rangle \\
= & \sum_{q>0} \frac{\pi}{q L}\left[-\sinh 2 \varphi_{q}+\cosh 2 \varphi_{q} \cdot \cos 2 q x\right. \\
& +i \sin 2 q x], \\
D_{2}(x)= & \langle 0|\phi(x) \phi(x)| 0\rangle \\
= & \sum_{q>0} \frac{\pi}{q L}\left[-\sinh 2 \varphi_{q} \cdot \cos 2 q x\right. \\
& \left.+\cosh 2 \varphi_{q}\right], \quad D_{1}(x)-D_{2}(x) \\
= & \sum_{q>0} \frac{\pi}{q L} \frac{q}{\sqrt{m^{2}+q^{2}}}(\cos 2 q x-1) \\
& +\sum_{q>0} \frac{\pi}{q L} \cdot i \sin 2 q x .
\end{aligned}
$$

Using the following mathematical formulas

$$
\begin{gathered}
\int_{0}^{\infty} d q \frac{\cos 2 q x}{\sqrt{m^{2}+q^{2}}}=K_{0}(2 m x), \\
-\sum_{q=n \pi / L, n>0} \frac{e^{-a q}}{\sqrt{m^{2}+q^{2}}}=\gamma+\ln \frac{m a}{2}+O\left(\frac{1}{L}\right), \\
\sum_{q>0} \frac{\pi}{q L} e^{i q z-\alpha z}=\ln \frac{L}{\pi \alpha}+\ln \frac{\alpha}{|z|}+\frac{i \pi}{2} \operatorname{sgn}(z),
\end{gathered}
$$

where $K_{0}$ is the modified Bessel function and $\gamma$ is the Euler constant, we find

$$
\frac{-1}{2 \pi a} e^{D_{1}(x)-D_{2}(x)}=\frac{m}{4 \pi} e^{\gamma} \cdot e^{K_{0}(2 m x)}
$$

Putting everything together, we finally get

$$
\langle\bar{\psi}(x) \psi(x)\rangle=\frac{m}{2 \pi} e^{\gamma} \cdot e^{K_{0}(2 m x)} .
$$

The above formula is obtained for $L \rightarrow \infty$. The right edge of the segment at $L$ is pushed away to infinity, so we have effectively the behavior of the condensate on a half-line. When $x$ is near the origin, the condensate becomes singular because of the chiral symmetry breaking boundary condition and the exact correlation of $\psi_{L}$ and $\psi_{R}$ at the origin. In regions far away from the origin, we expect that the condensate is not affected by the boundary condition. Indeed, when $x \gg m, K_{0}(m x) \rightarrow 0$ and $\bar{\psi}(x) \psi(x) \rightarrow(m / 2 \pi) e^{\gamma}$ which is just the usual chiral condensate on an infinite line as it should be [3,5-9]. When $x$ is very near the edge, the condensate diverges as $1 / x$. This behavior of the condensate near the boundary is also found by Dürr [15].

The above result can be readily generalized to the finite temperature case. One only need to substitute $\left\langle\hat{b}_{q}^{\dagger} \hat{b}_{q}\right\rangle=1$ $-\left\langle\hat{b}_{q} \hat{b}_{q}^{\dagger}\right\rangle=n(T) \equiv 1 /\left(e^{\omega(q)}-1\right)$ into Eq. (14). Here $\omega(q)$ $=\sqrt{m^{2}+q^{2}}$ is the energy dispersion for the boson excitations. Upon doing that, we have

$$
\begin{aligned}
D_{1}(q)-D_{2}(q)= & \sum_{q>0}\left\{[1+2 n(T)]\left(\cosh 2 \varphi_{q}+\sinh 2 \varphi_{q}\right)\right. \\
& \times(\cos 2 q x-1)+i \sin 2 q x\}
\end{aligned}
$$

and the chiral condensate at finite temperature becomes

$$
\begin{aligned}
\langle\bar{\psi}(x) \psi(x)\rangle_{T>0}= & \langle\bar{\psi}(x) \psi(x)\rangle_{T=0} \\
& \times \exp \sum_{q>0} \frac{\pi}{L} \frac{2}{e^{\sqrt{m^{2}+q^{2}} / T}-1} \frac{\cos 2 q x}{\sqrt{m^{2}+q^{2}}} \\
& \times \exp \sum_{q>0} \frac{\pi}{L} \frac{-2}{e^{\sqrt{m^{2}+q^{2}} / T}-1} \frac{1}{\sqrt{m^{2}+q^{2}}} .
\end{aligned}
$$


For $m x \gg 1$, the second factor approaches 1 and the above result again coincides with the one obtained in the whole line case. Equations (18) and (20) are the main results of this paper. Inclusion of a finite chemical potential is also straightforward following a previous treatment for the standard case [10].

\section{DISCUSSIONS}

It is important to point out that because of the symmetry breaking boundary condition, a unique ground state has been selected among the usual degenerate vacua far away from the boundary. The analogous situation in the usual spin system with a boundary is familiar: If we impose the condition that spins on the boundary must all point to the same direction, say up, the spin system will settle down to a state with spins all pointing up when temperature lower than the critical temperature is approaching zero; one unique ground state is selected. If we rotate the boundary spin, the orientation of the spins deep inside will also change accordingly. Likewise here, if we change the boundary condition to $\psi_{R}(t, 0)=$ $-e^{i \theta} \psi_{L}(t, 0)$, the chiral condensates Eqs. (18) and (20), will contain an extra factor $\cos (\theta)$ where $\theta$ is the famous vacuum angle. To understand this, one only needs to look at Eq. (13) and see that an extra factor $e^{i \theta}$ must be put on the right-hand side. The $\cos (\theta)$ factor arises from combining $e^{i \theta}$ with $e^{-i \theta}$ that will thereby appear in the Hermitian conjugate of Eq. (13).

Finally, we note that for the free massless fermion case, i.e., charge $e=0$, the chiral condensate can be easily calculated from Eq. (13) to be proportional to $1 / 2 \pi x$ when $L$ $\rightarrow \infty$. As a check, we can also obtain this result by taking the limit $m(=e / \sqrt{\pi}) \rightarrow 0$ in Eq. (18).

Summing up, we have demonstrated that the chiral condensate can be obtained exactly in the Schwinger model on a half-line if we choose an appropriate (yet not unphysical) boundary condition. More general boundary conditions may not allow exact treatments.

\section{ACKNOWLEDGMENTS}

This work is supported by the National Science Council of Taiwan under grant NSC 89-2112-M-002-056.
[1] J. Schwinger, Phys. Rev. 128, 2425 (1962).

[2] J.H. Loewenstein and J.A. Swieca, Ann. Phys. (N.Y.) 68, 172 (1961).

[3] N.S. Manton, Ann. Phys. (N.Y.) 159, 220 (1985).

[4] E. Abdalla, M. C. B. Abdalla, and K. D. Rothe, Nonperturbative Methods in Two-Dimensional Quantum Field Theories (World Scientific, Singapore, 1991).

[5] N.K. Nelson and B. Schroer, Nucl. Phys. B210, 62 (1977).

[6] Y.-C. Kao, Mod. Phys. Lett. A 7, 1411 (1992).

[7] I. Sachs and A. Wipf, Helv. Phys. Acta 65, 652 (1992).

[8] A.V. Smilga, Phys. Lett. B 278, 371 (1992).

[9] J.E. Hetrick and Y. Hosotani, Phys. Rev. D 38, 2621 (1988); J.E. Hetrick, Y. Hosotani, and S. Iso, ibid. 53, 7255 (1996).

[10] Y.-C. Kao and Y.W. Lee, Phys. Rev. D 50, 1165 (1994). The unusual oscillatory behavior in the chiral condensate when chemical potential is not zero is due to the momentum anomaly explained by Manton in Ref. [3], p. 229.
[11] V.A. Rubakov, Nucl. Phys. B203, 311 (1982).

[12] C. Callan, Phys. Rev. D 25, 2141 (1982).

[13] I. Affleck and J. Sagi, Nucl. Phys. B417, 413 (1994); J. Polchinski, ibid. B242, 345 (1984).

[14] Y.-C. Kao, Phys. Lett. B143, 147 (1984).

[15] Stephan Dürr, Ann. Phys. (N.Y.) 273, 1 (1999).

[16] Stephan Dürr and Andreas Wipf, Ann. Phys. (N.Y.) 255, 333 (1997).

[17] This boundary condition on $A_{0}$ will allow us to avoid nonessential infrared problems.

[18] S. Eggert and I. Affleck, Phys. Rev. B 46, 10866 (1992); M. Fabrizio and A. Gogolin, ibid. 51, 17827 (1995).

[19] J. Polchinski and L. Thorlacius, Phys. Rev. D 50, R622 (1994).

[20] For a concise review of bosonization technique, see also R. Shankar, in Lectures given at the BCSPIN School, Katmandu, 1991, Condensed Matter and Particle Physics, edited by Y. Lu, J. Pati, and Q. Shafi (World Scientific, Singapore, 1993). 